\title{
ORBITS IN A STÄCKEL APPROXIMATION
}

\author{
V. DE BRUYNE, F. LEEUWIN AND H. DEJONGHE \\ Sterrenkundig Observatorium, Universiteit Gent, Belgium
}

Because of their analytical simplicity and regularity, Stäckel potentials are attractive tools for modelling galaxies. The third integral $I_{3}$ is explicitly known in a Stäckel potential, and can be used as an approximation to the effective third integral, in order to construct three-integral models (cf. Dejonghe, et al. , 1996, A\&A 306, 363).

Moreover, Stäckel potentials turn out to yield good global descriptions for either axisymmetric or triaxial systems without central mass concentration (de Zeeuw 1985, MNRAS 216, 273, de Zeeuw \& Lynden-Bell 1985, MNRAS 215, 713), and even for some systems with a black hole included (Sridhar \& Touma 1997, MNRAS 292, 657).

One long-standing concern though, is that Stäckel potentials form only a very small subspace in the family of all potentials. The main orbit families found by numerical integration in general triaxial potentials are present in a Stäckel potential (Schwarzschild 1981, ApJ 232, 236, de Zeeuw 1985, MNRAS 216, 273), but there is obviously no place in an integrable potential for smaller orbital families or stochastic orbits. However, since regular orbits are the rule rather than the exception, a potential which yields a good representation of those orbits is certainly a good basis for building models.

We want to improve the generality of models based on Stäckel potentials, by using a set of Stäckel potentials, each of which fits the true galactic potential in a spatially limited region. These potentials then provide an explicit expression for the integrals that will allow us to construct semi-analytical distribution functions expressed as $f\left(E, L_{z}, I_{3}\right)$. In a Stäckel potential, there is a function of 1 variable that can be freely chosen. This flexibility is advantageous, and will, of course, be exploited at the fullest when performing the fit.

This work is a preliminary study on the feasibility and effectiveness of such an approach. This we do by comparing orbit integrations with their counterparts in the Stäckel-set representation.

As a test case, we consider an axisymmetric Miyamoto-Nagai (MN) model (1975, PASJ 27, 533) with intermediate flattening $(\epsilon \sim 4.5)$. In this model, the diskiness is largely exaggerated, so we are considering a specially demanding case. The fitting of Stäckel potentials to the MN potential in spatially limited regions is done on a grid, using quadratic programming. In brief, a number of basic potentials $\psi_{i}$ are chosen out of a library and combined to yield a $\psi_{S}=\sum_{i} c_{i} \psi_{i}$, in order to minimize the quantity $\chi^{2}=\sum_{l}\left(\Psi-\sum_{i} c_{i} \psi_{i}\right)^{2}$, with $l$ an index covering the points in the grid. A complete description of this fit method can be found in Mathieu \& Dejonghe, 1996, A\&A 314, 25. The integration of orbits in both potentials, is performed using a fourth-order Runge-Kutta with variable time-step 
and with energy conservation better than $10^{-6}$ (relative error) over 100 azimuthal periods.

Checking the representation of orbits in both potentials, we find that typical orbits have very similar surfaces of section. Minor resonances trapping some of the orbits are well reproduced by the Stäckel potentials. Other resonances present in the MN potential, but absent from the Stäckel fit, are found for small values of $I_{3}$ (these are orbits remaining close to the equatorial plane, where diskiness is important). They represent $\sim 4 \%$ of our orbit library. Those orbits could be represented by an alternative method (such as a frequency decomposition).

The volume of phase-space occupied by the orbit is important when assembling orbits to reproduce a given density. Therefore, we also computed the orbital densities $\rho\left(R, z ; E, L_{z}, I_{3}\right)$ which are functions of $(R, z)$ for each given orbit. The mass fraction correctly located in the Stäckel potential compared to the original MN potential is $M=1-\delta M$, where we computed $\delta M=\sum_{k, \ell} \mid\left(\rho_{M N}\left(R_{k}, z_{\ell}\right)-\right.$ $\left.\rho_{S}\left(R_{k}, z_{\ell}\right)\right) \mid / 2$. The average $M$ is $92 \%$, the lowest values for $M$ are found for orbits with resonances that were not well fitted.

An important question is how well the $I_{3}$ is conserved along the orbits, so that it can be used as a label in modelling procedures. We calculated the variation of $I_{3}$ along the orbits and find that it is usually of order the error in the potential fit (at worst a few percents). For orbits that remain close to the equatorial plane, the variations reach a few tenths. This shows that, for a real galaxy, a strong diskiness would certainly require an additional local Stäckel potential for these regions.

We can conclude that, using a small set of Stäckel potentials as an approximation to a trial MN potential, we are able to reproduce most of its orbits with satisfactory accuracy, except for very few resonances. We find that for the vast majority of orbits, the Stäckel $I_{3}$ does provide a valid approximation for the effective third integral of our trial potential. Therefore, this value of $I_{3}$ can be used for labelling those orbits when constructing dynamical models. 